\title{
HEDGING IN FINANCIAL MARKETS ${ }^{1}$
}

\author{
By MARTIN BAXTER \\ Statistical Laboratory, Cambridge University
}

\begin{abstract}
This (mostly) expository paper describes the importance of hedging to the pricing of modern financial products and how hedging may be achieved even when the traditional Black-Scholes assumptions are absent.
\end{abstract}

\section{KEYWORDS}

Derivatives; hedging; option-pricing; superhedging; volatility.

\section{OVERVIEW}

Any market practitioner who sells derivatives on his own account will say that hedging is the key to pricing. If a contract is not hedged, one can sell it at any price, even the right one, and still lose money. The price of the contract must be the cost of the hedge, plus margin, and the profit/loss of the deal will depend crucially on the hedge being effective.

From the earliest days of the rigorous literature, such as Harrison and Pliska (1981), hedging has been used to derive prices in the absence of arbitrage. Text books for practitioners, such as Chapter 14 of Hull (1997) and Baxter and Rennie (1996) stress the centrality of hedging to securities trading. The essence of the case being that hedging allows the derivative writer to minimise his exposure to market risk without reducing his profit, thus allowing him, in the words of one banker, 'to quote a price with a view of making a profit through his intermediation rather than by taking a directional view' (Bogni, 1997).

Hedging may be performed on a wide variety of markets for arbitrary derivative products. In simple cases, an option is hedged by trading in the underlying security (stock, currency or bond), but it is equally possible to construct a hedge for a derivative in terms of simpler derivatives, such as forwards and calls. Dupire (1993) has done interesting work in developing this area of option-hedging, which we will study in section 5 .

On the other hand, it might be attractive to work with a model in which it is not possible to hedge. Such incomplete markets can appear intractable, but, following work by El Karoui et al (1996) are now amenable to the tool of

\footnotetext{
I This paper was delivered at a meeting on 'Financial Mathematics and Derivatives' at the International Centre for Mathematical Sciences in Edinburgh on 21 January 1997.
}

ASTIN BULLETIN, Vol. 28, No. 1, 1998, pp. 5-16 
superhedging. This technique produces a strategy that dominates the option payoff. That is, the hedge will produce at least as much as the contract requires, and may produce a surplus. Eliminating down-side market risk (in theory at least) is achieved at the expense of the loss of two-way pricing. Finally, the superhedging of derivatives using other options gives even better results, and creates an elegant duality between the option-hedging and the superhedging approaches.

\section{STATIC HEDGING}

We begin with the simplest case.

Consider the contract to forward purchase at time $T$ one unit of a stock $S$ for a pre-set price $k$. Imagine that interest rates are constant at a (continuously compounded) rate $r$ and there are no transaction costs payable nor dividends due from the stock. At what price $k$ should we sell the forward contract?

At time $T$, the contract has the (now certain) value of

$$
X=S_{T}-k,
$$

so we might expect its time-zero discounted worth to be

$$
\mathbb{E}\left(e^{-r T} X\right)=e^{-r T} \mathbb{E}\left(S_{T}\right)-k e^{-r T},
$$

and then the price $k$ required to give the contract nil net present value would be $k=\mathbb{E}\left(S_{T}\right)$. More generally, we might discount equities at a different rate, $u$, than the cash discount rate $r$. In that case, the appropriate forward price would be $k=e^{-(u-r) T} \mathbb{E}\left(S_{T}\right)$. Either way, this seems to make some sense: if $S_{T}$ is expected to be large, the forward price should be correspondingly large. Paradoxically however, this price is wrong.

The actual forward price, in this model, is $k=e^{r T} S_{0}$. That is, the price is just the current stock price $S_{0}$ scaled up by the time value of money over the period. The price does not depend at all on whether $S_{T}$ is expected to be high or low. The reason for this is a hedge. The contract $X$ can be hedged if we:

- buy one unit of stock for price $S_{0}$, and

- borrow $k e^{-r T}$ units of cash.

This has initial cost $S_{0}-k e^{-r T}$. By time $T$, the stock has evolved to be worth $S_{T}$ and the debt has grown to $-k$, giving exactly the same net worth as the forward $X$. So the initial worth of $X$ is the initial cost of the hedge, which is zero only if $k=e^{r T} S_{0}$.

The hedge is essentially to buy one unit of the stock and wait, so that it is ready to be handed over at time $T$. We are unconcerned whether the stock price rises or falls, or indeed whether it is valued 'correctly' at either time 0 or time $T$. It is enough for us to have it, because we are now unexposed to market risk, in the form of stock price movements.

This example demonstrates a static hedge, which can be put on at the start of the contract and left unchanged till the end. 
Example: forward borrowing The interest-rate market can be described through the behaviour of zero-coupon discount bonds. The $T$-bond pays one unit of cash at time $T$, and at time $t$ before then has a value (typically) less than 1 , written $P(t, T)$. This allows us to lend $£ 1$ to a customer from time zero to time $T$ by selling $P^{-1}(0, T)$ units of the $T$-bond into the market now for a price of $£ 1$, which we loan to the customer. At time $T$, the customer pays us back $P^{-1}(0, T)$ which we use to meet our maturing $T$-bond liability. We could also accept term deposits from the customer, by changing all the signs and buying $T$-bonds instead.

Our customer may wish instead to borrow later (from time $S$ to time $T$ ), but agree on the price now, at time zero. Suppose he wants to borrow $£ 1$ at time $S$. How much should we demand back at time $T$ ?

The answer is, we should get back $P(0, S) / P(0, T)$ and here is the hedge:

- sell $P(0, S) / P(0, T)$ units of $T$-bond, and receive $P(0, S)$ now, and

- buy one unit of $S$-bond, for cost $P(0, S)$ now.

These initial transactions have zero net cost. At time $S$, we receive $£ 1$ from our $S$-bond which we can loan to the customer as agreed. At time $T$, we receive $P(0, S) / P(0, T)$ from the customer which exactly cancels our maturing $T$-bond liability.

In other words, the forward price to sell the $T$-bond at time $S$ is

$$
F=\frac{P(0, T)}{P(0, S)}
$$

Away from the special case of forwards, static hedging can still be beneficial, even if it is not perfect. For instance, a static hedge to approximate a claim $X$ can be made by holding $\phi$ units of stock and $\psi$ units of the cash bond. The expected square error of this hedge (to choose a simple loss function), is

$$
\mathbb{E}\left(\left(X-\phi S_{T}-\psi e^{r T}\right)^{2}\right)
$$

We can minimise this, to begin with, over the cash holding, with the optimal choice of $\psi$ being $\psi=e^{-r T} \mathbb{E}\left(X-\phi S_{T}\right)$, and the minimal value being $E(\phi)=\operatorname{Var}\left(X-\phi S_{T}\right)$. This itself can now be minimised over $\phi$ at the value

$$
\phi=\frac{\operatorname{Cov}\left(X, S_{T}\right)}{\operatorname{Var}\left(S_{T}\right)}
$$

with value $E(\phi)=\operatorname{Var}(\mathrm{X})\left(1-\rho^{2}\right)$, where $\rho$ is the correlation between $X$ and $S_{T}$.

Example In the particular case where $S_{T}$ is normally distributed as a $N\left(\mu, \sigma^{2}\right)$ and $X$ is the call payoff $X=\left(S_{T}-\mu\right)^{+}$, then the optimal $\phi=\frac{1}{2}$, and

$$
E(\phi)=\frac{\pi-2}{2 \pi-2} E(0)
$$

a reduction in the error variance of over $73 \%$. 


\section{SIMPLE HEDGING}

In a sense, forwards are a special case and their hedge has been known for a long time. In fact, any payoff which is a linear function of the stock price has an exact static hedge. The hedge for the claim $X=a S_{T}+b$, where $a$ and $b$ are constants, is to hold $a$ units of stock and $e^{-r T} b$ units of cash, with initial value $a S_{0}+e^{-r T} b$.

This exact answer for simple claims in general markets also holds for general claims in simple markets. For instance, take the single-period market with zero interest rates (so there is a constant cash bond $B_{t}=1$ ) and one risky asset $S_{t}$. The stock evolves as shown in figure 1.

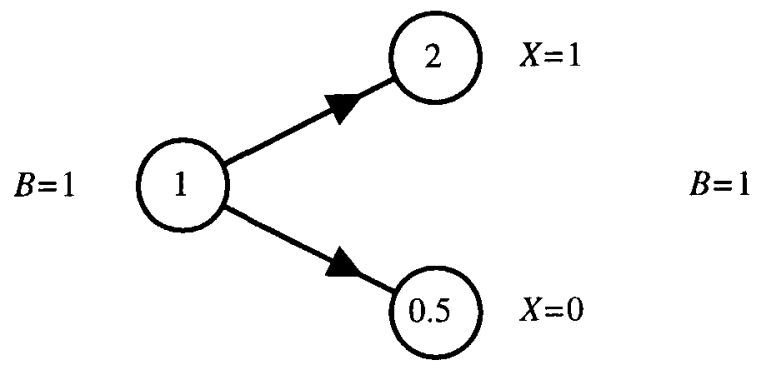

Figure 1: Single-period securities market

The stock price either doubles or halves, and cash stays constant at 1 . A call option on $S_{1}$, struck at $£ 1$, with time 1 value of $X=\left(S_{1}-1\right)^{+}$, will pay off $£ 1$ if the stock goes up and nothing if it goes down. The paucity of possible values for $S_{1}$ enables us to write $X$ as

$$
X=\frac{2}{3} S_{1}-\frac{1}{3}
$$

That is, $X$ has the same payoff as a forward to buy $2 / 3$ units of stock at the price of $£ 0.50$ per unit. Applying our methods of section 2 , we see that the time zero price for $X$ is

$$
V=\frac{2}{3} S_{0}-\frac{1}{3}=\frac{1}{3}
$$

So the price of $X$ is actually $1 / 3$ and the hedge is to

- buy $2 / 3$ units of stock for cost $2 / 3$

- borrow an additional $1 / 3$ units of cash, which has initial cost of $1 / 3$ and terminal value of $X$.

We could have performed this calculation for any claim $X$ which paid $x_{u}$ after an up-jump and $x_{d}$ after a down-jump. Such a claim would be worth

$$
V=\frac{1}{3} x_{u}+\frac{2}{3} x_{d}
$$


This has the form of the expected value of $X$ under a probability measure which assigns $1 / 3$ chance to an up-jump and $2 / 3$ chance to a down-jump. This hedging measure $(q, 1-q)$ is given by the formula

$$
q=\frac{S_{0}-s_{d}}{s_{u}-s_{d}}, \text { or } q=\frac{e^{r \delta t} S_{0}-s_{d}}{s_{u}-s_{d}} \text { if } r \neq 0,
$$

where $S_{1}$ takes the value $s_{u}$ after an up-jump, and $s_{d}$ after a down-jump. To see why this actually is an expectation, see Chapter 2 of Baxter and Rennie (1996).

Although the model is very simple, it can be used as a basic building block of more complex models. We can combine many individual branches into a tree (figure 2).

It just takes 10 layers in this tree to produce a final layer containing over 1000 nodes. Options can still be priced by working back recursively through the tree from the final layer. See, for example, Chapter 15 of Hull (1997) or Chapter 2 of Baxter and Rennie (1996).

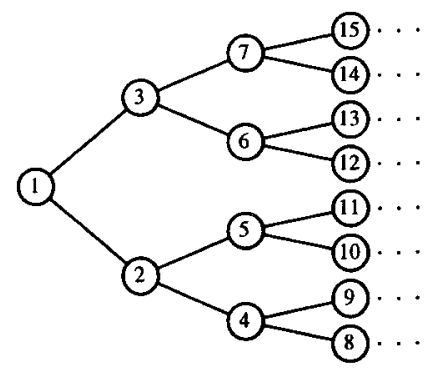

Figure 2: Binomial tree

\section{BLACK-SCHOLES}

The simplest continuous-time model for a stock price is the Black-Scholes model,

$$
S_{t}=S_{0} \exp \left(\sigma W_{t}+\mu t\right)
$$

where $W_{l}$ is a Brownian motion, and $\sigma$ and $\mu$ are constants. In this model, $\log \left(S_{t} / S_{0}\right)$ is normally distributed with variance $\sigma^{2} t$ and mean $\mu_{t}$. The variable $\sigma$ is called the volatility of the process.

We can also see $S$ as the limit of discrete trees, as in section 3, with current value $S_{0}$ evolving to

$$
S_{\delta t}=\left\{\begin{array}{cl}
S_{0} \exp (\sigma \sqrt{\delta t}+\mu \delta t) & \text { if up-jump } \\
S_{0} \exp (-\sigma \sqrt{\delta t}+\mu \delta t) & \text { if down-jump. }
\end{array}\right.
$$


The time increment $\delta t$ over one step of the tree is going to get ever smaller. Then $\log \left(S_{t} / S_{0}\right)$ is equal to $\mu t+\sigma \sqrt{\delta t} X(t / \delta t)$, where $X_{n}$ is a simple symmetric random walk, with $X_{n}$ distributed as a function of a binomial, $2 \operatorname{Bin}(n, 1 / 2)-n$, with zero mean and variance $n$. By the Central Limit theorem, the distribution of $\log \left(S_{t} / S_{0}\right)$ converges to that of the Black-Scholes, namely $N\left(\mu t, \sigma^{2} t\right)$.

Choosing $q$ to be the hedging measure

$$
q=\frac{e^{r \delta t}-e^{-\sigma \sqrt{\delta t}+\mu \delta t}}{e^{\sigma \sqrt{\delta t}+\mu \delta t}-e^{-\sigma \sqrt{\delta t}+\mu \delta t}} \sim \frac{1}{2}\left(1-\sqrt{\delta t}\left(\frac{\mu+\frac{1}{2} \sigma^{2}-r}{\sigma}\right)\right),
$$

then now $\log \left(S_{t} / S_{0}\right)$ is asymptotically distributed as a normal $N\left(\left(r-\frac{1}{2} \sigma^{2}\right) t, \sigma^{2} t\right)$. The hedging price at time zero of any claim $X$ payable at time $T$ will then be $\mathbb{E}_{\mathbb{Q}}\left(e^{-r T} X\right)$, where the behaviour of $X$ under $\mathbb{Q}$ is governed by the new asymptotic normal distribution. Evaluating this for the European call claim $X=\left(S_{T}-k\right)^{+}$ gives rise to the celebrated Black and Scholes (1973) call option pricing formula

$$
V_{0}=e^{-r T}\left(F \Phi\left(\frac{\log \frac{F}{k}+\frac{1}{2} \sigma^{2} T}{\sigma \sqrt{T}}\right)-k \Phi\left(\frac{\log \frac{F}{k}-\frac{1}{2} \sigma^{2} T}{\sigma \sqrt{T}}\right)\right),
$$

where $F$ is the forward price $F=S_{0} e^{r T}$, and $\Phi$ is the normal distribution function.

Again we do not use this price because it is an expected value of the claim, but because this is the value which lets us hedge. In this case the hedge we need at time 0 is $\frac{\partial V}{\partial S}$. For a general option $X$ we can also price and hedge in the same way.

In fact the Black-Scholes formula is not just true for the Black-Scholes model. It is enough that the stock $S_{T}$ and cash bond $B_{T}$ are jointly log-normally distributed under the hedging measure $\mathbb{Q}$. The formula will then still hold with $\sigma^{2} T$ replaced by $\operatorname{Var}\left(\log \left(S_{T} / S_{0}\right)\right), e^{-r T}$ replaced by $\mathbb{E}_{\mathbb{Q}}\left(B_{T}^{-1}\right)$, and the forward price $F$ equal to $F=S_{0} / \mathbb{E}_{\mathbb{Q}}\left(B_{T}^{-1}\right)$.

For a good introduction to Black-Scholes from the actuarial point of view, see the comprehensive review paper by Kemp (1996).

Also Hobson (1996a) reviews the extensions possible from the constant volatility assumptions of the basic Black-Scholes model. That paper describes hedging in a stochastic volatility framework, as well as considering discrete-time ARCH and GARCH models, and provides a good introduction to the more advanced techniques.

\section{OPTION-HEDGING}

The above formula does depends crucially on some aspects of the Black-Scholes model, namely that

- volatility is constant (or at least deterministic)

- the market generated by the asset is complete

In practice, these can not be relied upon. One solution is to recognise that, say, vanilla call options are so frequently traded as to be liquid assets in their own right. As such, they are not priced per se by the Black-Scholes formula, but they 
can themselves be used in hedges to price more complicated products. This is, to coin a phrase, option-hedging: using options as a hedge for other derivatives, as opposed to the classical hedging of options.

As an example, suppose we have a traded stock $S_{t}$ and traded call options, where $C_{l}(T, y)$ is the time $t$ price of a call on $S_{T}$ struck at $y$. For simplicity, take interest rates to be zero, so that

$$
C_{t}(T, y)=\mathbb{E}_{\mathbb{Q}}\left(\left(S_{T}-y\right)^{+} \mid \mathcal{F}_{t}\right) .
$$

A particular case, originally due to Breeden and Litzenberger (1978), is that of a terminal value payoff $X=f\left(S_{T}\right)$, for some twice-differentiable function $f$. A simple variant of Taylor's theorem says that

$$
f(x)=f(0)+x f^{\prime}(0)+\int_{0}^{\infty}(x-y)^{+} f^{\prime \prime}(y) d y, \text { for al } x \geq 0
$$

which can be proved by integrating by parts. Substituting $S_{T}$ for $x$ and taking expectations under $\mathbb{Q}$ gives the time $t$ value of the option, $V_{t}$, as

$$
V_{t}=f(0)+S_{t} f^{\prime}(0)+\int_{0}^{\infty} C_{t}(T, y) f^{\prime \prime}(y) d y .
$$

We have calculated not only the price for $X$, but also a static hedge which is

- hold $f(0)$ units of cash,

- hold $f^{\prime}(0)$ units of stock, and

- hold $f^{\prime \prime}(y) d y$ units of the call struck at $y$.

(In practice, some approximation to the continuous density $f^{\prime \prime}(y) d y$ will be required.) We have already seen how linear terms can be statically hedged like a forward. Now we see the convex terms being hedged with options.

If interest rates were non-zero, the formula still holds with the single change that we hold $e^{-r T} f(0)$ units of the cash bond, which is worth $e^{-r(T-t)} f(0)$ at time $t$.

Note that this does not price all options, such as lookbacks or exotics. (For example, a put at the maximum price attained by the stock, $X=\sup _{t<T} S_{t}-S_{T}$; or an down-and-out call which only pays off if the stock never went below a preset threshold, $X=\left(S_{T}-k\right)^{+} I\left(\inf _{t \leq T} S_{t}>c\right)$.)

The formula's advantages are not only that it is a static hedge, but also that it is completely model independent: we make no assumptions about how $S_{t}$ or $C_{t}(T, k)$ evolve, or even whether the market is complete. But we can still hedge.

This example is actually evidence of a deeper idea of Dupire (1993). Given the option prices

$$
C_{t}(T, y)=\mathbb{E}_{\mathbb{Q}}\left(\left(S_{T}-y\right)^{+} \mid \mathcal{F}_{t}\right)
$$

their partial derivative with respect to $y$ is

$$
\frac{\partial}{\partial y} C_{t}(T, y)=-\mathbb{Q}\left(S_{T}>y \mid \mathcal{F}_{t}\right)
$$


and differentiating once more gives

$$
\frac{\partial^{2}}{\partial y^{2}} C_{t}(T, y) d y=\mathbb{Q}\left(S_{T} \in d y \mid \mathcal{F}_{t}\right)
$$

which is the marginal density of $S_{T}$ given $\mathcal{F}_{t}$. Then the time $t$ value of any claim $X=f\left(S_{T}\right)$ will be

$$
\int_{0}^{\infty} \mathbb{Q}\left(S_{T} \in d y \mid \mathcal{F}_{t}\right) f(y)=\int_{0}^{\infty} \frac{\partial^{2}}{\partial y^{2}} C_{t}(T, y) f(y) d y .
$$

Integration by parts transforms this into the Breeden and Litzenberger formula.

\section{Convex payoffs}

There is a special case of terminal-value options with a convex payoff, which is particularly interesting. For instance, we can use Jensen's inequality (see, for example, 6.6 of Williams, 1991) to show that

$$
V_{0}=\mathbb{E}_{\mathbb{Q}}\left(e^{-r T} f\left(S_{T}\right)\right) \geq e^{-r T} f(F),
$$

where $F$ is a forward price $F=e^{r T} S_{0}=\mathbb{E}_{\mathbb{Q}}\left(S_{T}\right)$. We can also use the convexity of $f$ once more to show that

$$
V_{0} \geq e^{-r T} f(F) \geq f\left(S_{0}\right)-\left(1-e^{-r T}\right) f(0) .
$$

So that if $f(0)=0$, for perhaps a call option, the option value $V_{0}$ is always worth at least as much as its current intrinsic value $f\left(S_{0}\right)$, and similarly $V_{t} \geq f\left(S_{t}\right)$. American options, which give the right to the intrinsic value $f\left(S_{t}\right)$ at any time $t$ up to maturity $T$, have no additional worth for such convex payoffs null at 0 .

We can also see how volatility and convexity make prices higher. The price of a convex option is increasing in the volatility of the asset. For instance, if

$$
S_{T}^{\sigma}=F \exp \left(\sigma Z-\frac{1}{2} \sigma^{2}\right)
$$

where $Z$ is a normal $N(0,1)$, giving $\mathbb{E}\left(S_{T}^{\sigma}\right)=\mathrm{F}$, then for $\sigma^{2}<\tau^{2}$,

$$
S_{T}^{\tau}=S_{T}^{\sigma} \exp \left(\alpha \tilde{Z}-\frac{1}{2} \alpha^{2}\right) \text { for } \tilde{Z} \text {, an independent } N(0,1),
$$

where $\alpha^{2}=\tau^{2}-\sigma^{2}$. Then again by Jensen's inequality

$$
\mathbb{E}\left(f\left(S_{T}^{\tau}\right)\right)=\mathbb{E}\left(\mathbb{E}\left(f\left(S_{T}^{\tau}\right) \mid S_{T}^{\sigma}\right)\right) \geq \mathbb{E}\left(f\left(S_{T}^{\sigma}\right)\right)
$$

Call prices, for instance, increase with the volatility of the asset (as per the BlackScholes formula), but also in general models. This fact, coupled with the Breeden and Litzenberger formula, shows how volatility and convexity work together to give value. That is, the option's non-linear terms have worth

$$
\int_{0}^{\infty} C_{I}(T, y) f^{\prime \prime}(y) d y
$$


which increases both with the volatility of the asset (which increases all the call prices) and the convexity of $f$ (which increases $f^{\prime \prime}$ ).

Hobson (1996b) unites existing results, using coupling, to show that convexoption prices, even for diffusion models, increase with volatility and that the option value is itself a convex function of the current asset price.

A new result generalises the Breeden and Litzenberger formula to higher dimensions. Suppose we have a vector of assets $S_{t}$ in $\mathbb{R}^{\mathrm{n}}$, such that $\left|S_{T}\right|$ is squareintegrable and that options on all fixed portfolios (linear combinations) of $S_{T}$ are traded. That is, the call $\left(\left\langle\theta, S_{T}\right\rangle-y\right)^{+}$is traded, for all vectors $\theta$ in $\mathbb{R}^{\mathbf{n}}$ and all real $y$, and has current price $C_{t}(T, \theta, y)$.

Now for any $f$ in $C^{n+3}$, which satisfies the boundedness condition that $\left|\nabla^{n+3} f\right|$ is integrable over $\mathbb{R}^{\mathrm{n}}$, then $f$ has a Fourier transform $\tilde{f}(\theta)$,

$$
\tilde{f}(\theta)=\int_{\mathbb{R}^{\mathrm{n}}} e^{-i\langle\theta, x\rangle} \tilde{f}(x) d x
$$

which is bounded by $|\tilde{f}(\theta)| \leq c|\theta|^{-(n+3)}$, for some constant $c$. We recall the Fourier inversion formula

$$
f(x)=(2 \pi)^{-n} \int_{\mathbb{R}^{\mathrm{n}}} e^{i(\theta, x)} \tilde{f}(\theta) d \theta .
$$

We can also use an adapted version of the existing one-dimensional hedging representation applied to the complex function $e^{i z}$, evaluated at the portfolio value $z=\left\langle\theta, S_{T}\right\rangle$, thus:

$$
e^{i\left\langle\theta, S_{T}\right\rangle}=1+i\left\langle\theta, S_{T}\right\rangle-\int_{\mathbb{R}}|y|\left(y^{-1}\left\langle\theta, S_{T}\right\rangle-1\right)^{+} e^{i y} d y .
$$

We can substitute this expression into the Fourier inversion formula above, to deduce that

$$
f\left(S_{T}\right)=f(0)+\left\langle\nabla f(0), S_{T}\right\rangle-(2 \pi)^{-n} \int_{\mathbb{R}^{n}} \int_{\mathbb{R}}|y|\left(y^{-1}\left\langle\theta, S_{T}\right\rangle-1\right)^{+} e^{i y} \tilde{f}(\theta) d y d \theta .
$$

This expression can be re-expressed, by changing variables to $\phi=y^{-1} \theta$, to give

$$
f\left(S_{T}\right)=f(0)+\left\langle\nabla f(0), S_{T}\right\rangle+\int_{\mathbb{R}^{\mathrm{n}}}\left(\left\langle\phi, S_{T}\right\rangle-1\right)^{+} F_{f}(\phi) d \phi
$$

where $F_{f}(\phi)=-(2 \pi)^{-n} \operatorname{Re} \int_{\mathbb{R}}|y|^{n+1} e^{i y} \tilde{f}(y \phi) d y$. Thus the time $t$ price of such a claim $f\left(S_{T}\right)$ is

$$
V_{t}=f(0)+\left\langle\nabla f(0), S_{t}\right\rangle+\int_{\mathbb{R}^{n}} C_{t}(T, \phi) F_{f}(\phi) d \phi,
$$

where $C_{t}(T, \phi)=C_{t}(T, \phi, 1)$. And thus when our claim, $X=f\left(S_{T}\right)$, is the terminal-time evaluation of a smooth function $f$, then the claim has a static hedge 
of cash, stocks, and generalised calls. As such functions are dense in the space of all measurable functions $f$, with $f\left(S_{T}\right)$ integrable, then all such claims can be approximated with static hedges.

\section{SUPERHEDGING}

In incomplete models, where we cannot hedge, and we are pricing exotics (insusceptible to Breeden and Litzenberger), we must try something else. Work by El Karoui et al. (1996) has brought forward the concept of superhedging.

A clear treatment of the El Karoui results can be found in Hobson (1996b), and Frey (1997) is a good review of the current literature and developments in the superhedging field.

Suppose as an example, a stock price behaves under some martingale measure, as a martingale diffusion with volatility $\sigma_{t}$,

$$
d S_{t}=\sigma_{t} S_{t} d W_{t} .
$$

Suppose further that $\sigma_{t}$ is either dependent on a new source of randomness distinct from $W_{t}$ or simply uncertain - we just do not have a reliable model for it.

Given an upper bound $\sigma_{M}$ on the volatility, that is $\sigma_{t} \leq \sigma_{M}$, we can bound the price of convex terminal value claims $f\left(S_{T}\right)$. We can even allow $\sigma_{M}$ to be nonconstant, as long as $\sigma_{M}=\sigma_{M}\left(S_{t}, t\right)$ only depends on time and the current stock price. If we hedge as if the actual volatility is $\sigma_{M}$ then, as the theorem below shows, our hedge's final value will always be at least as large as $f\left(S_{T}\right)$. The claim has been superhedged. So the super-price of the claim is the theoretical price of $f\left(S_{T}\right)$ given the stock's volatility is $\sigma_{M}$. Similarly concave payoffs are superhedged by lower bounds on volatility.

THEOREM (El Karoui et al.) Let $C_{t}=C\left(S_{t}, t\right)$ be the worth of the convex claim $f\left(S_{T}\right)$ assuming the volatility is $\sigma_{M}$, and let $V_{t}$ be the worth of the attempted hedge. Then $C(x, t)$ is convex in $x$ and the tracking error $e_{t}=V_{t}-C_{t}$ is given by the positive quantity

$$
e_{t}=\frac{1}{2} \int_{0}^{t}\left(\sigma_{M}^{2}-\sigma_{u}^{2}\right) S_{u}^{2} \frac{\partial^{2} C}{\partial x^{2}}\left(S_{u}, u\right) d u .
$$

In the special case where $f(x)$ is the call payoff $(x-k)^{+}$, then $C_{t}=C\left(S_{t}, t\right)$ is the Black-Scholes call price, assuming constant volatility $\sigma_{M}$, where $C=C(x, t)$ is

$$
C(x, t)=x \Phi\left(\frac{\log \frac{x}{k}+\frac{1}{2} \sigma_{M}^{2}(T-t)}{\sigma_{M} \sqrt{T-t}}\right)-k \Phi\left(\frac{\log \frac{x}{k}-\frac{1}{2} \sigma_{M}^{2}(T-t)}{\sigma_{M} \sqrt{T-t}}\right),
$$

and the hedge $\phi_{t}$ is equal to $\phi_{t}=\frac{\partial C}{\partial x}\left(S_{t}, t\right)$, where

$$
\frac{\partial C}{\partial x}=\Phi\left(\frac{\log \frac{x}{k}+\frac{1}{2} \sigma_{M}^{2}(T-t)}{\sigma_{M} \sqrt{T-t}}\right)
$$


Then the worth of the hedge at time $t$ is $C_{0}+\int_{0}^{t} \phi_{u} d S_{u}$. Crucially, $C$ is convex in $x$ so that the tracking error is always positive as $\sigma_{M}$ is an upper bound for $\sigma_{t}$. At time $T, V_{T}$ is the worth of the hedge and $C_{T}$ is the option worth $\left(S_{T}-k\right)^{+}$, so a positive tracking error means the option has been superhedged.

A pleasing synthesis between the option-hedging of section 5 and superhedging has been achieved by Paras (1997), a description of which we close with.

Following Paras, we let $\mathcal{P}$ be the set of all measures that might model the asset price. These measures might not be equivalent, for instance they might correspond to all possible Markov volatility processes $\sigma_{t}$ lying in a band

$$
\sigma_{m} \leq \sigma_{t} \leq \sigma_{M}
$$

where $\sigma_{m}, \sigma_{M}$ can be functions of time and asset price. Then the superhedge price of a claim $X$ will be the (supermartingale) process

$$
V_{t}=\sup _{\mathbb{P} \in \mathcal{P}} \mathbb{E}_{\mathbb{P}}\left(X \mid \mathcal{F}_{t}\right)
$$

where interest rates have been set to zero for simplicity. The superhedging strategy will be to behave as if volatility is

$$
\sigma=\left\{\begin{array}{l}
\sigma_{M} \text { when } \frac{\partial^{2} \mathrm{~V}}{\partial \mathrm{S}^{2}}>0, \text { locally convex } \\
\sigma_{m} \text { when } \frac{\partial^{2} \mathrm{~V}}{\partial \mathrm{S}^{2}}>0, \text { locally concave }
\end{array}\right.
$$

Suppose also that there are currently traded instruments, such as vanilla options, which pay off $X_{i}$ at time $T$ and are currently worth $C_{i}(t)$. We might not be able to write $X$ entirely in terms of a combination of the $X_{i}$, but we could do the best we could. If we used a hedge of $\lambda_{i}$ units of $X_{i}$, our valuation for $X$ would be

$$
L_{l}(\lambda)=\sup _{\mathbb{P} \in \mathcal{P}} \mathbb{E}_{\mathbb{P}}\left(X-\sum_{i} \lambda_{i} X_{i} \mid \mathcal{F}_{t}\right)+\sum_{i} \lambda_{i} C_{i}(t) .
$$

As we are completely (super)-hedged for any choice of $\lambda$, we could choose $\lambda$ to minimise $L_{t}(\lambda)$, and quote the sharpest price possible. As $L_{t}(\lambda)$ is the supremum of linear functions of $\lambda$, it is a convex function of $\lambda$, and so susceptible to optimization techniques.

Interestingly, this problem is the Lagrangian dual of the constrained optimization problem which maximises the expectation of $X$ over measures which produce the market price for every $X_{i}$. That is the problem

$$
\sup \mathbb{E}_{\mathbb{P}}\left(X \mid \mathcal{F}_{t}\right) \text {, subject to } \mathbb{E}_{\mathbb{P}}\left(X_{i} \| \mathcal{F}_{\cdot t}\right)=C_{i}(t) ; \mathbb{P} \in \mathcal{P} \text {. }
$$

This is really just affirms the intuitive observation that measures in $\mathcal{P}$ which do not reflect the current price of traded instruments cannot be the measure we need to price. So we have a duality between the best superhedge of the claim over all measures, allowing hedging with traded instruments, and the best superhedge over all measures which price the traded instruments to market. 


\section{REFERENCES}

BAXter, M.W. and RenNie, A.J.O. (1996) Financial Calculus: an introduction to derivative pricing, Cambridge University Press.

BlaCk, F. and Scholes, M. (1973) The pricing of options and corporate liabilities, J. Political Econ., $81,637-654$.

Breeden, D.T. and Litzenberger, R.H. (1978) Price of state contingent claims implicit in options prices, J. Business, 51, 621-651.

Bogni, R. (1997) Don't forget your tool kit, Times Higher Ed., 24/01/97, p28.

DUPIRE, B. (1993) Arbitrage pricing with stochastic volatility, SORTresearch paper, Paribas Capital Markets.

El Karoui, N., JeanblanC-Picqué, M. and Shreve, S. (1996) Robustness of the Black and Scholes formula, preprint.

FREY, R. (1997) Derivative asset analysis in models with level-dependent and stochastic volatility, CWI Quarterly (to appear).

HARrison, J.M. and PliskA, S.R. (1981) Martingales and stochastic integrals in the theory of continuous trading, Stoch. Procs. and their applications, 11, 215-260.

Hobson, D.G. (1996a) Stochastic Volatility, to appear in Statistics in Finance, ed. by D. Hand and S. Jacka, Edward Arnold.

HoBson, D.G. (1996b) Robust hedging via coupling, University of Bath preprint, School of Mathematics 96:04.

Hull, J. (1997) Options, futures and other derivative securities, third edition, Prentice-Hall.

KEMP, M.H.D. (1997) Actuaries and derivatives, to appear in British Actuarial Journal.

PARAS, A. (1997) Hedging under uncertain volatility, SORTresearchpaper, Paribas Capital Markets.

Williams, D. (1991) Probability with Martingales, Cambridge University Press.

MARTIN BAXTeR

Statistical Laboratory

Cambridge University

16 Mill Lane

Cambridge CB2 ISB

England 